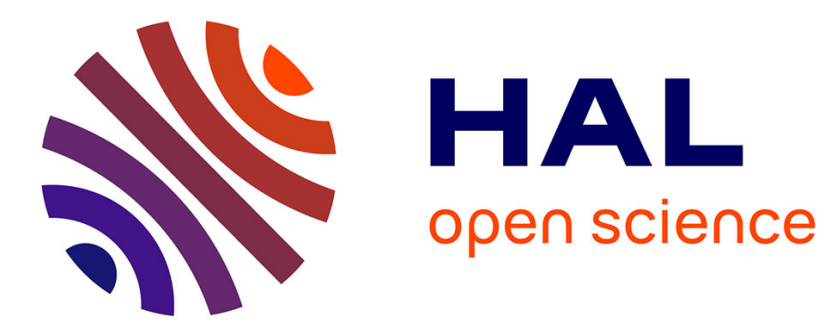

\title{
Minimum-Density Identifying Codes in Square Grids
}

\author{
Marwane Bouznif, Frédéric Havet, Myriam Preissmann
}

\section{To cite this version:}

Marwane Bouznif, Frédéric Havet, Myriam Preissmann. Minimum-Density Identifying Codes in Square Grids. 11th International Conference, AAIM 2016, Riccardo Dondi, Jul 2016, Bergamo, Italy. pp.77-88, 10.1007/978-3-319-41168-2_7. hal-01346750

\section{HAL Id: hal-01346750 \\ https://hal.inria.fr/hal-01346750}

Submitted on 19 Jul 2016

HAL is a multi-disciplinary open access archive for the deposit and dissemination of scientific research documents, whether they are published or not. The documents may come from teaching and research institutions in France or abroad, or from public or private research centers.
L'archive ouverte pluridisciplinaire HAL, est destinée au dépôt et à la diffusion de documents scientifiques de niveau recherche, publiés ou non, émanant des établissements d'enseignement et de recherche français ou étrangers, des laboratoires publics ou privés. 


\title{
Minimum-density identifying codes in square grids
}

\author{
Marwane Bouznif ${ }^{1}$, Frédéric Havet ${ }^{2}$, and Myriam Preissmann ${ }^{3}$ \\ 1 A-SIS, Saint Étienne, France. (email:marwane.bouznif@a-sis.com) \\ 2 Projet COATI, I3S (CNRS, UNSA) and INRIA, Sophia Antipolis. Partly \\ supported by ANR Blanc STINT. (email:Frederic.Havet@sophia.inria.fr) \\ 3 Univ. Grenoble Alpes and CNRS, G-SCOP, Grenoble, France. \\ (email:myriam.preissmann@grenoble-inp.fr)
}

\begin{abstract}
An identifying code in a graph $G$ is a subset of vertices with the property that for each vertex $v \in V(G)$, the collection of elements of $C$ at distance at most 1 from $v$ is non-empty and distinct from the collection of any other vertex. We consider the minimum density $d^{*}\left(\mathcal{S}_{k}\right)$ of an identifying code in the square grid $\mathcal{S}_{k}$ of height $k$ (i.e. with vertex set $\mathbb{Z} \times\{1, \ldots, k\})$. Using the Discharging Method, we prove $\frac{7}{20}+\frac{1}{20 k} \leq$ $d^{*}\left(\mathcal{S}_{k}\right) \leq \min \left\{\frac{2}{5}, \frac{7}{20}+\frac{3}{10 k}\right\}$, and $d^{*}\left(\mathcal{S}_{3}\right)=\frac{7}{18}$.
\end{abstract}

Keywords: identifying code, square grid, discharging method

\section{Introduction}

The two-way infinite path, denoted $P_{\mathbb{Z}}$, is the graph with vertex set $\mathbb{Z}$ and edge set $\{\{i, i+1\}: i \in \mathbb{Z}\}$. For every positive integer $k$, the finite path of length $k-1$, denoted $P_{k}$, is the subgraph of $P_{\mathbb{Z}}$ induced by $\{1,2, \ldots, k\}$.

The cartesian product of two graphs $G$ and $H$, denoted by $G \square H$, is the graph with vertex set $V(G) \times V(H)$ and edge set $\{(a, x)(b, y) \mid$ either $(a=b$ and $x y \in E(H))$ or $(a b \in E(G)$ and $x=y)\}$. A square grid is the cartesian product of two paths, which can be finite or infinite. The square lattice is the cartesian product $P_{\mathbb{Z}} \square P_{\mathbb{Z}}$ of two two-way infinite paths and is denoted by $\mathcal{G}$. For every positive integer $k$, we denote by $\mathcal{S}_{k}$ the square grid $P_{\mathbb{Z}} \square P_{k}$

Let $G$ be a graph. The closed neighbourhood of $v$, denoted $N[v]$, is the set of vertices that are either $v$ or adjacent to $v$ in $G$. A set $C \subseteq V(G)$ is an identifying code in $G$ if for every vertex $v \in V(G), N[v] \cap C \neq \emptyset$, and for any two distinct vertices $u, v \in V(G), N[u] \cap C \neq N[v] \cap C$.

Let $G$ be a (finite or infinite) graph. For any non-negative integer $r$ and vertex $v$, we denote by $B_{r}(v)$ the ball of radius $r$ in $G$, that is $B_{r}(v)=\{x \mid$ $\operatorname{dist}(v, x) \leq r\}$. For any set of vertices $C \subseteq V(G)$, the density of $C$ in $G$, denoted by $d(C, G)$, is defined by

$$
d(C, G)=\limsup _{r \rightarrow+\infty} \frac{\left|C \cap B_{r}\left(v_{0}\right)\right|}{\left|B_{r}\left(v_{0}\right)\right|},
$$


where $v_{0}$ is an arbitrary vertex in $G$. The infimum of the density of an identifying code in $G$ is denoted by $d^{*}(G)$. Observe that if $G$ is finite, then $d^{*}(G)=$ $\left|C^{*}\right| /|V(G)|$, where $C^{*}$ is a minimum-size identifying code in $G$.

The problem of finding identifying codes of small density was introduced in [10] in relation to fault diagnosis in arrays of processors. Identifying codes are also used in [11] to model a location detection problem with sensor networks. Identifying codes of the grids have been studied [2], [5], [9], [10] as well as variations where instead of considering the closed neighbourhood to identify a vertex, the the ball of radius $r$ (for some fixed $r$ ) is considered [2], [8]. The closely related problem of finding a locating-dominating set with minimum density has also been studied [12].

In this paper, we are interested in identifying codes of square grids, and more specifically the $\mathcal{S}_{k}$. The tile depicted in Figure 1 was given in [3]. It generates a

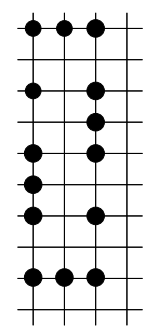

Fig. 1. Tile generating an optimal identifying code of the grid.

periodic tiling of the plane with periods $(0,10)$ and $(4,1)$, yielding an identifying code $C_{\mathcal{G}}^{*}$ of the square lattice with density $\frac{7}{20}$. Ben-Haim and Litsyn [1] proved that this density is optimal, that is $d^{*}(\mathcal{G})=\frac{7}{20}$.

Daniel, Gravier, and Moncel [6] showed that $d^{*}\left(\mathcal{S}_{1}\right)=\frac{1}{2}$ and $d^{*}\left(\mathcal{S}_{2}\right)=\frac{3}{7}$. For larger value of $k$, they proved the following lower and upper bound on $d^{*}\left(\mathcal{S}_{k}\right)$ : $\frac{7}{20}-\frac{1}{2 k} \leq d^{*}\left(\mathcal{S}_{k}\right) \leq \min \left\{\frac{2}{5}, \frac{7}{20}+\frac{2}{k}\right\}$. In this paper, we improve on both the lower and upper bounds of $d^{*}\left(\mathcal{S}_{k}\right)$. We prove

$$
\frac{7}{20}+\frac{1}{20 k} \leq d^{*}\left(\mathcal{S}_{k}\right) \leq \min \left\{\frac{2}{5}, \frac{7}{20}+\frac{3}{10 k}\right\} .
$$

The upper bound is obtain by deriving an identifying code of $\mathcal{S}_{k}$ with density $\frac{7}{20}+\frac{3}{10 k}$ from the optimal identifying code $C_{\mathcal{G}}^{*}$ of the square lattice.

The lower bound is obtained using the Discharging Method and proceeds in two phases. The first one is a rewriting of the proof of Ben-Haim and Litsyn [1] as a Discharging Method proof. Doing so, it becomes clear that it extends to any square grid, and so that $d^{*}(G) \geq \frac{7}{20}$ for any square grid. It makes it also possible to improve on this bound when $G=\mathcal{S}_{k}$ with $k \geq 3$ in a second phase. 
We strongly believe that both our upper and lower bounds may be improved using the same general techniques. In fact, to obtain the upper bound, we only alter the code $C_{\mathcal{G}}^{*}$ on the top two rows and the bottom two rows of $\mathcal{S}_{k}$. Looking for alterations on more rows, possibly with the help of a computer, will certainly yield codes with smaller density. We made no attempt to optimize the second phase in the lower bound proof. Doing more complicated discharging rules, based on more complicated properties of identifying codes will surely give better bounds. However, we do not see any way to make the two bounds meet for all $k$. Nevertheless, we are able to do it for $k=3$ : we show that $d^{*}\left(\mathcal{S}_{3}\right)=\frac{7}{18}$.

\section{General upper bounds}

Theorem 1. For all $k \geq 7$, we have $d^{*}\left(\mathcal{S}_{k}\right) \leq \frac{7}{20}+\frac{3}{10 k}$.

Proof. Let $K_{k}$ be the code of $\mathcal{S}_{k}$ obtained from $C_{\mathcal{G}}^{*}$ on $\mathcal{S}_{k}$ by replacing the rows $\mathbb{Z} \times\{1\}$ and $\mathbb{Z} \times\{2\}$ by the rows depicted in Figure 2 and the rows $\mathbb{Z} \times\{k-1\}$ and $\mathbb{Z} \times\{k\}$ by the ones obtained symmetrically.

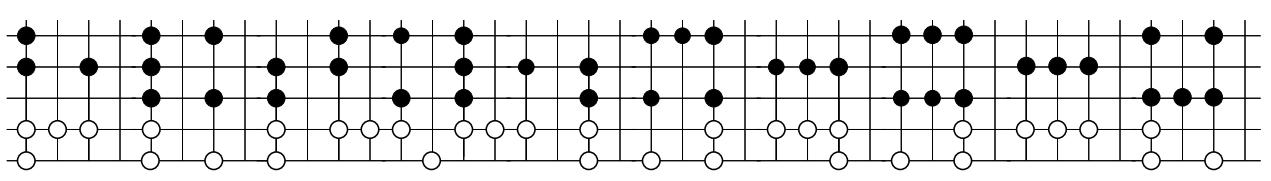

Fig. 2. The bottom rows (white disks) of $K_{k}$.

We claim the code $K_{k}$ is identifying. Indeed since $C_{\mathcal{G}}^{*}$ is an identifying code of the square lattice, it suffices to check that for every vertex $v \in \mathbb{Z} \times\{1,2,3, k-$ $2, k-1, k\}$, there is no vertex $w$ such that $N[v] \cap K_{k}=N[w] \cap K_{k}$. This can be easily done.

The density of $K_{k}$ on $\mathbb{Z} \times\{1,2, k, k-1\}$ is $\frac{34}{80}$. So, the density of $K_{k}$ is $\frac{7}{20}\left(1-\frac{4}{k}\right)+\frac{34}{80} \times \frac{4}{k}=\frac{7}{20}+\frac{3}{10 k}$.

\section{Lower bounds on $d^{*}\left(S_{k}\right)$}

The aim of this section is to show that $d^{*}\left(\mathcal{S}_{k}\right) \geq \frac{7}{20}+\frac{1}{20 k}$.

The general idea is to consider an identifying code $C$ in a square grid $G$. We assign an initial weight $w(v)$ to each vertex where $w(v)=1$ if $v \in C$ and $w(v)=0$ otherwise. We then apply some local discharging rules. In such rules, some vertices send part of their weight to some other vertices at distance at most $s$, for some fixed integer $s$. We then prove that the final weight $w^{*}(v)$ of every vertex $v$ is at least $d^{*}$. We claim that it implies $d(C, G) \geq d^{*}$. This is trivial if $G$ is bounded. Suppose now that $G=\mathcal{S}_{k}$. Since a vertex sends at most 1 to 
vertices at distance at most $s$, a charge of at most $\left|B_{r+s}\left(v_{0}\right) \backslash B_{r}\left(v_{0}\right)\right| \leq 2 s k$ enters $B_{r}\left(v_{0}\right)$ during the discharging phase. Thus

$\left|C \cap B_{r}\left(v_{0}\right)\right|=\sum_{v \in B_{r}\left(v_{0}\right)} w(v) \geq \sum_{v \in B_{r}\left(v_{0}\right)} w^{*}(v)-\left|B_{r+s}\left(v_{0}\right) \backslash B_{r}\left(v_{0}\right)\right| \geq d^{*} \cdot\left|B_{r}\left(v_{0}\right)\right|-2 s k$.

But $\left|B_{r}\left(v_{0}\right)\right| \geq(2 r+1) k-k^{2}$, thus $d\left(C, \mathcal{S}_{k}\right) \geq \limsup _{r \rightarrow+\infty}\left(d^{*}-\frac{2 s k}{(2 r+1) k-k^{2}}\right)=$ $d^{*}$. This proves our claim. We then deduce $d^{*}\left(S_{k}\right) \geq d^{*}$.

Let $C$ be an identifying code in a square grid $G$. We denote by $U$ the set of vertices not in $C$. For $1 \leq i \leq 5$, we define $L_{i}=\{v \in G|| N[v] \cap C \mid=i\}$, and we set $C_{i}=L_{i} \cap C$ and $U_{i}=L_{i} \cap U$. Observe that $U_{5}$ is empty. For $X \in\{C, L, U\}$ we set $X_{\geq i}=\bigcup_{j=i}^{5} X_{j} \quad$ and $\quad X_{\leq i}=\bigcup_{j=1}^{i} X_{j}$.

For every set $S \subseteq V(G)$, a vertex in $S$ is called an $S$-vertex.

The following proposition is a direct consequence of the definition of identifying code.

Proposition 1. Let $C$ be an identifying code in a square grid $G$.

(i) Every vertex in $C$ has at most one neighbour in $U_{1}$.

(ii) Every vertex in $C_{1}$ has no neighbour in $U_{1}$.

(iii) Two vertices in $C_{2}$ are not adjacent.

Let $C^{\prime}$ be the set of vertices in $C_{1}$ that have four neighbours in $G$ that belong all to $U_{\leq 2}$. Let $\tilde{L}_{3}$ be the set of vertices in $C_{3}$ having at least one neighbour in $C_{\geq 3}$. Set $\bar{L}_{3}=L_{3} \backslash \tilde{L}_{3}$ and $\bar{C}_{3}=C_{3} \backslash \tilde{L}_{3}$

Proposition 2 (Ben-Haim and Litsyn [1]). Let $C$ be an identifying code in a square grid $G$. There is a bipartite graph $H$ with bipartition $\left(C^{\prime}, L_{\geq 3}\right)$ such that

(i) the degree of every element of $C^{\prime}$ is at least 4 ,

(ii) the degree of every element of $\bar{L}_{3}$ is at most 2 ,

(iii) the degree of every element of $\tilde{L}_{3}$ is at most 6 , and

(iii) the degree of every element of $L_{\geq 4}$ is at most 4.

Proof. Ben-Haim and Litsyn [1] proved Proposition 2 for another definition of the set $\tilde{L}_{3}$ : their set is larger than ours. However it happens that using word for word the same rules as Ben-Haim and Litsyn (Steps 1 to 10 in [1]) for building the bipartite graph from an identifying code of the square lattice, regardless to the fact that $\tilde{L}_{3}$ is not the same set, we get a bipartite graph that may be different but has exactly the same degree properties ; the proof of this fact is exactly the same as the one of [1].

Furthermore, in the construction of Ben-Haim and Litsyn, the neighbours in $H$ of an element $c^{\prime}$ of $C^{\prime}$ are always in the rectangle with corners $c^{\prime}$ and another vertex $c \in C$. Therefore it is in any square grid containing those two vertices, and so their proof works for any square grid. However, it might be possible that this vertex is not in $G$ if this graph is not a square grid and the proof of Proposition 2 does not work for any induced subgraph $G$ of $\mathcal{G}$. 
Remark 1. The graph $H$ in Proposition 2 may have some double edges.

Theorem 2. Let $G$ be a square grid. Then $d^{*}(G) \geq \frac{7}{20}$.

Proof. Let $C$ be an indentifying code in $G$ and $H$ be a bipartite graph associated to $C$ as described in Proposition 2. We give an initial weight 1 to the vertices of $C$ and 0 to the vertices in $U$. We then apply the following discharging rules, one after another. So if several rules must be applied to a same vertex, then it will send charge several times.

(R1) Every vertex of $C$ sends $\frac{7}{20}$ to each neighbour in $U_{1}$ and $\frac{7}{40}$ to each neighbour in $U_{\geq 2}$.

(R2) Every vertex of $L_{\geq 3}$ sends $\frac{1}{20}$ to its neighbours in $C_{\leq 2}$.

(R3) Every vertex of $L_{\geq 3}$ sends $\frac{1}{80}$ to each $C^{\prime}$-vertex to which it is adjacent in $H$ by one edge and $\frac{2}{80}$ to each $C^{\prime}$-vertex to which it is adjacent in $H$ by two edges.

Let us prove that the final weight $w^{\prime}(v)$ of each vertex $v$ is at least $7 / 20$.

If $v \in C^{\prime}$, then its original weight is 1 . By Proposition 1-(ii), it has no $U_{1}$ neighbour. Hence it sends $\frac{7}{40}$ to each of its four neighbours in $U$ by $(R 1)$, and receives $\frac{1}{80}$ from each of its at least four edges in $H$ by (R3). Hence $w^{\prime}(v) \geq$ $1-4 \cdot \frac{7}{40}+4 \cdot \frac{1}{80}=\frac{7}{20}$.

If $v \in C_{1} \backslash C^{\prime}$, then its original weight is 1 . By Proposition 1-(ii), it has no $U_{1}$ neighbour. By definition of $C^{\prime}$, it has a neighbour in $L_{\geq 3}$ from which it receives $\frac{1}{20}$ by $(R 2)$. Hence $w^{\prime}(v) \geq 1-4 \cdot \frac{7}{40}+\frac{1}{20}=\frac{7}{20}$.

If $v \in U_{1} \cup U_{2}$, then its original weight is 0 . It receives $\frac{7}{20}$ by (R1), and it does not send anything. Hence $w^{\prime}(v)=\frac{7}{20}$.

If $v \in C_{2}$, then its original weight is 1 . By Proposition 1-(i), it has at most one $U_{1}$ neighbour, so its sends at most $\frac{7}{20}+2 \cdot \frac{7}{40}$ by (R1). Moreover, by Proposition 1(iii), it has a neighbour in $C_{\geq 3}$ from which it receives $\frac{1}{20}$ by (R2). Hence $w^{\prime}(v) \geq$ $1-\frac{7}{20}-2 \cdot \frac{7}{40}+\frac{1}{20}=\frac{7}{20}$.

If $v \in \tilde{L}_{3}$, then it is in $C_{3}$, so its original weight is 1. By Proposition 1-(i), it has at most one $U_{1}$ neighbour, so it sends at most $\frac{7}{20}+\frac{7}{40}$ by (R1). By definition of $\tilde{L}_{3}, v$ has at most one neighbour in $C_{\leq 2}$, so its sends at most $\frac{1}{20}$ by (R2). Finally, it has degree at most 6 in $H$, so it sends at most $6 \cdot \frac{1}{80}$ by (R3). Hence $w^{\prime}(v) \geq 1-\frac{7}{20}-\frac{7}{40}-\frac{1}{20}-6 \cdot \frac{1}{80}=\frac{7}{20}$.

If $v \in \bar{C}_{3}$, then it is in $C_{3}$, so its original weight is 1. By Proposition 1(i), it has at most one $U_{1}$ neighbour, so it sends at most $\frac{7}{20}+\frac{7}{40}$ by (R1). It has at most two neighbours in $C_{\leq 2}$, so its sends at most $2 \cdot \frac{1}{20}$ by (R2). And it has degree at most 2 in $H$, so it sends at most $2 \cdot \frac{1}{80}$ by (R3). Hence $w^{\prime}(v) \geq 1-\frac{7}{20}-\frac{7}{40}-2 \cdot \frac{1}{20}-2 \cdot \frac{1}{80}=\frac{7}{20}$.

If $v \in U_{3}$, then its original weight is 0 . It receives $3 \cdot \frac{7}{40}$ by (R1). It has at most three neighbours in $C_{\leq 2}$, so its sends at most $3 \cdot \frac{1}{20}$ by (R2). And it has degree at most 2 in $H$, so it sends at most $2 \cdot \frac{1}{80}$ by (R3). Hence $w^{\prime}(v) \geq$ $3 \cdot \frac{7}{40}-3 \cdot \frac{1}{20}-2 \cdot \frac{1}{80}=\frac{7}{20}$.

If $v \in C_{4}$, then its original weight is 1 . It send at most $\frac{7}{20}$ to its unique $U$-neighbour by (R1). It has at most three neighbours in $C_{\leq 2}$, so its sends at 
most $3 \cdot \frac{1}{20}$ by $(\mathrm{R} 2)$. And it has degree at most 4 in $H$, so it sends at most $4 \cdot \frac{1}{80}$ by $(\mathrm{R} 3)$. Hence $w^{\prime}(v) \geq 1-\frac{7}{20}-3 \cdot \frac{1}{20}-4 \cdot \frac{1}{80}=\frac{9}{20}$.

If $v \in U_{4}$, then its original weight is 0 . It receives $4 \cdot \frac{7}{40}$ by (R1). It has at most four neighbours in $C_{\leq 2}$, so its sends at most $4 \cdot \frac{1}{20}$ by (R2). And it has degree at most 4 in $H$, so it sends at most $4 \cdot \frac{1}{80}$ by (R3). Hence $w^{\prime}(v) \geq$ $4 \cdot \frac{7}{40}-4 \cdot \frac{1}{20}-4 \cdot \frac{1}{80}=\frac{9}{20}$.

If $v \in C_{5}$, then its original weight is 1 . It has no $U$-neighbour. It has at most four neighbours in $C_{\leq 2}$, so its sends at most $4 \cdot \frac{1}{20}$ by (R2). And it has degree at most 4 in $H$, so it sends at most $4 \cdot \frac{1}{80}$ by $(\mathrm{R} 3)$. Hence $w^{\prime}(v) \geq 1-4 \cdot \frac{1}{20}-4 \cdot \frac{1}{80}=\frac{15}{20}$.

Thus at the end, $w^{\prime}(v) \geq \frac{7}{20}$ for all vertex $v$, so $d(C, G) \geq \frac{7}{20}$.

Theorem 2 is tight because $d^{*}(\mathcal{G})=\frac{7}{20}$. However, for $\mathcal{S}_{k}$, we can improve on $7 / 20$.

Theorem 3. For any $k \geq 3, d^{*}\left(\mathcal{S}_{k}\right)=\frac{7}{20}+\frac{1}{20 k}$.

Proof. Let us first give some definition. In $\mathcal{S}_{k}$, the row of index $i$, denoted $R_{i}$, is the set of vertices $\mathbb{Z} \times\{i\}$, the column of index $j$, denoted $Q_{j}$, is the set of vertices $\{j\} \times\{1, \ldots, k\}$. The border vertices are those of $R_{1} \cup R_{k}$.

Let $C$ be an identifying code in $\mathcal{S}_{k}$.

We first apply the discharging phase as in the proof of Theorem 2. At the end of this phase every vertex has weight at least $7 / 20$. But some of them may have a larger weight.

It is for example the case of $C_{4}$-vertices which have weight at least $9 / 20$. Let $D_{3}$ be the set of vertices of $C_{3}$ having no neighbour in $C_{\leq 2}$. Observe that $D_{3} \subseteq \tilde{L}_{3}$. A vertex of $D_{3}$ do not send anything by (R2), hence its weight is at least $\frac{8}{20}$. Set $D=D_{3} \cup C_{4}$.

Consider also border $C$-vertices. Such vertices are missing one neighbour, so for any $1 \leq i \leq 4$, border $C_{i}$-vertices gives to one $U$-neighbour less than non-border $C_{i}$-vertices by $\left(R_{1}\right)$. It follows that if $v$ is a border $C$-vertex, then $w(v) \geq \frac{7}{20}+\frac{7}{40}$.

The following claim shows that there are many vertices in $R_{1} \cup R_{2}$ with a weight larger than $7 / 20$.

Claim. Let $C$ be a code of $\mathcal{S}_{k}$. If $\{(a-3,1),(a-2,1),(a-1,1),(a, 1),(a+$ $1,1),(a+2,1),(a+3,1)\} \cap C=\emptyset$, then $(a, 2)$ is in $D$.

Proof. If $\{(a-3,1),(a-2,1),(a-1,1),(a, 1),(a+1,1),(a+2,1),(a+3,1)\} \cap C=\emptyset$, then necessarily $(a-2,2),(a-1,2),(a, 2),(a+1,2)$, and $(a+2,2)$ are in $C$, because each vertex has a neighbour in $C$. Therefore $(a-1,2),(a, 2)$, and $(a+1,2)$ are in $C_{\geq 3}$ and so $(a, 2)$ is in $D$.

We then proceed to a second discharging phase. Set $S_{j}=Q_{j-3} \cup Q_{j-2} \cup$ $Q_{j-1} \cup Q_{j} \cup Q_{j+1} \cup Q_{j+2} \cup Q_{j+3}$.

(R4) Every vertex in $D$ gives $\frac{1}{20 k}$ to every vertex in its column.

(R5) Every border $C$-vertex in column $Q_{j}$ gives $\frac{1}{40 k}$ to every vertex in $S_{j}$. 
Let us examine the weight $w^{*}(v)$ of a vertex $v$ after this phase.

Observe first that every vertex receives at least $\frac{1}{20 k}$ during this second phase. Indeed, if $v=(a, b)$ has a $D$-vertex in its column, then it receives $\frac{1}{20 k}$ from it by $(\mathrm{R} 4)$. If it has no $D$-vertex in its column, then by Claim 3 , a vertex in $\{(a-3,1),(a-2,1),(a-1,1),(a, 1),(a+1,1),(a+2,1),(a+3,1)\}$ is a border $C$-vertex, and symmetrically, a vertex in $\left\{\left(a_{3}, k\right),(a-2, k),(a-1, k),(a, k),(a+\right.$ $1, k),(a+2, k),(a+3, k)\}$ is a border $C$-vertex. And these two vertices send $\frac{1}{40 k}$ each to $v$ by $(\mathrm{R} 5)$, so $v$ receives at least $\frac{1}{20 k}$ in total.

If $v \in D$, then $w^{\prime}(v) \geq \frac{8}{20}$. By $(\mathrm{R} 4)$, it sends $\frac{1}{20 k}$ to the $k$ vertices of its column. Hence it sends $\frac{1}{20}$. Since it received at least $\frac{1}{20 k}, w^{*}(v) \geq \frac{7}{20}+\frac{1}{20 k}$.

If $v$ is a border $C$-vertex, then $w^{\prime}(v) \geq \frac{7}{20}+\frac{7}{40}$. By (R4), it sends $\frac{1}{40 k}$ to the $7 k$ vertices of $S_{j}$. Hence it sends $\frac{7}{40}$. It also receives at least $\frac{1}{20 k}$. So $w^{*}(v) \geq \frac{7}{20}+\frac{1}{20 k}$.

If $v$ is neither a border $C$-vertex, nor a $D$-vertex, then it does not send anything. So $w^{*}(v) \geq w^{\prime}(v)+\frac{1}{20 k} \geq \frac{7}{20}+\frac{1}{20 k}$.

To conclude, after the second phase, each vertex has weight at least $\frac{7}{20}+\frac{1}{20 k}$. Thus $d\left(C, \mathcal{S}_{k}\right) \geq \frac{7}{20}+\frac{1}{20 k}$.

\section{Optimal identifying code in $\mathcal{S}_{3}$.}

Theorem 4. $d^{*}\left(\mathcal{S}_{3}\right)=\frac{7}{18}$.

It is straightforward to check that repeating the tile of Figure 3 with period $(12,0)$, we obtain an identifying code of density $\frac{7}{18}$.

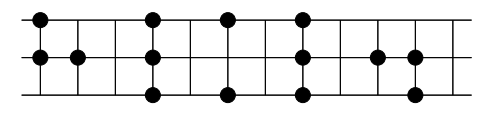

Fig. 3. Tile of a minimum-density identifying code in $\mathcal{S}_{3}$.

It remains to show that every identifying code in $\mathcal{S}_{3}$ has density at least $\frac{7}{18}$. We again use the Discharging Method: the technical details are more complicated than in the previous section, but the general framework is the same.

\subsection{Properties of codes in $\mathcal{S}_{3}$}

The lower row, (resp. central row, upper row) of $\mathcal{S}_{3}$, is the set of vertices in $\mathbb{Z} \times\{1\}$, (resp. $\mathbb{Z} \times\{2\}, \mathbb{Z} \times\{3\}$ ). A border vertex is a vertex on the upper or lower row. A central vertex is a vertex on the central row. The column of index $a$ is the set $\{(a, 1),(a, 2),(a, 3)\}$.

For convenience, instead of using the set $C_{i}$ we use the set $B_{i}$, which is defined as follows. A vertex is in $B_{i}$ if it is in $C$ and adjacent to $i$ vertices in $U$. Hence, a border vertex in $B_{i}$ is in $C_{4-i}$ and a central vertex in $B_{i}$ is in $C_{5-i}$.

Similarly to Proposition 1 , we get the following proposition. 
Proposition 3. Let $C$ be an identifying code in $\mathcal{S}_{3}$. Every border $B_{3}$-vertex has no neighbour in $U_{1}$.

Proposition 4. Let $C$ be an identifying code in $\mathcal{S}_{3}$. Every vertex in $B_{4}$ has a neighbour in $U_{\geq 3}$.

Proof. Let $x$ be a vertex in $B_{4}$. Necessarily $x$ must be a central vertex, that is, $x=(a, 2)$ for some $a$. Assume for a contradiction that $x$ has no neighbour in $U_{\geq 3}$. Then by Proposition 1 , its four neighbours are in $U_{2}$. Consider $u=(a, 3)$ : one of its neighbours $y$ is in $C$. By symmetry, we may assume $y=(a-1,3)$. Now the two vertices $u$ and $v=(a-1,2)$ are both adjacent to $x$ and $y$. Hence, since $u$ and $v$ are in $U_{2}$, we obtain $N[u] \cap C=\{x, y\}=N[v] \cap C$, a contradiction.

Proposition 5. Let $C$ be an identifying code in $\mathcal{S}_{3}$. Every border $C$-vertex adjacent to a central $B_{3}$ is in $B_{0} \cup B_{1}$.

Proof. Assume for a contradiction that a border $B_{2}$-vertex $y$ is adjacent to a central $B_{3}$-vertex $x$. Then $N[x] \cap C=N[y] \cap C=\{x, y\}$, a contradiction.

\subsection{Establishing the lower bound}

We use the Discharging Method. Let $C$ be an indentifying code in $\mathcal{S}_{3}$. We give an initial weight 1 to the vertices of $C$ and 0 to the vertices in $U$. We will then apply some discharging rules. Our aim is to prove that at the end the final weight of each vertex will be at least $\frac{7}{18}$.

For sake of clarity and to simplify the proof, we will perform these discharging rules in two stages.

A generous vertex is either a $B_{0}$-vertex or a border vertex in $B_{1}$ having its central neighbour in $C$. We first apply the following rules.

(R0) For $1 \leq i \leq 4$, every vertex of $C$ gives $\frac{7}{18 \times i}$ to each of its neighbours in $U_{i}$.

(R1) Every generous vertex gives $\frac{3}{18}$ to its central neighbour(s).

Let us denote by $w_{1}(v)$ the weight of the vertex $v$ after applying (R0-R1).

Observe that after (R0-R1) all the vertices of $U$ have weight exactly $\frac{7}{18}$. Indeed for all $1 \leq i \leq 4$, every vertex $u$ in $U_{i}$ receives $\frac{7}{18 \times i}$ from each of its $i$ neighbours. Hence in total it receives $\frac{7}{18}$ and so $w_{1}(u)=\frac{7}{18}$.

The weight of the vertices of $U$ will not change anymore and the charge will now only move from $C$-vertices to other $C$-vertices.

We define the excess of a vertex $v$ of $C$ as $\epsilon(v)=w_{1}(v)-\frac{7}{18}$. Informally, if it is positive, the excess of $v$ measures how much weight $v$ has above $\frac{7}{18}$ and thus can give to other vertices. If it is negative, the excess of $v$ measures the quantity of weight $v$ must receives from others to get weight $\frac{7}{18}$.

Observe that $B_{0^{-}}, B_{1^{-}}, B_{2^{-}}$and border $B_{3^{-}}$vertices have positive excess:

- If $v \in B_{0}$, then it gives nothing to $U$-vertices, and it gives $\frac{3}{18}$ to each (at most two) central neighbours. Thus $\epsilon(v) \geq 1-\frac{7}{18}-\frac{6}{18}=\frac{5}{18}$. 
- If $v \in B_{1}$, then it gives at most $\frac{7}{18}$ to its $U$-neighbour. So if it is not generous, $\epsilon(v) \geq 1-\frac{7}{18}-\frac{7}{18}=\frac{2}{9}$ and if $v$ is generous, $\epsilon(v) \geq 1-\frac{7}{18}-\frac{7}{18}-\frac{3}{18}=\frac{1}{18}$.

- If $v \in B_{2}$, then it is adjacent to at most one $U_{1}$-vertex by Proposition 1 . Hence $\epsilon(v) \geq 1-\frac{7}{18}-\frac{7}{18}-\frac{7}{36}=\frac{1}{36}$.

- If $v$ is a border $B_{3}$-vertex, then by Proposition 3 , it is adajcent to no $U_{1^{-}}$vertex. Hence it gives at most $\frac{7}{36}$ to each of its $U$-neighbours. So $\epsilon(v) \geq$ $1-\frac{7}{18}-3 \times \frac{7}{36}=\frac{1}{36}$.

On the opposite, some vertices of $B_{3} \cup B_{4}$ may have negative excess. Such vertices of $B_{3} \cup B_{4}$ will be called defective. Observe that defective vertices are on the central line. Moreover it is easy to check that a defective vertex has no generous neighbour. Indeed if a defective vertex $x$ has a generous neighbour $y$, then it is in $B_{3}$. Since $x$ has at most one $U_{1}$-neighbour, it sends at most $\frac{7}{18}+2 \times \frac{7}{36}=\frac{14}{18}$ to its $U$-neighbours. But it also receives $\frac{3}{18}$ from its generous neighbour. Hence $\epsilon(x) \geq 1-\frac{7}{18}-\frac{14}{18}+\frac{3}{18}=0$.

Simple calculations and Propositions 1, 3 and 4 show that a defective vertex is of one of the following kinds:

- a $B_{4}$-vertex with at least two $U_{2}$-neighbours;

- a central $B_{3}$-vertex with one $U_{1}$-neighbour and no generous neighbour.

We will now apply some new discharging rules in order to give charge to the defective vertices so that the final excess $\epsilon^{*}(v)$ of every vertex $v$ is non-negative. The rules are applied one after another, so if several rules must be applied to a same vertex then it will send charge several times.

For $S \in\{C, U\}$, an $S$-column is a column all vertices of which are in $S$. A right barrier (resp. left barrier) is a $C$-column such that the right (resp. left) neighbours of its two border vertices are in $U_{1}$. A lonely barrier is a barrier such that the columns to its right and its left are $U$-columns. Let $x$ be a $C$-vertex. Its right pal (resp. left pal) is the closest central $C$-vertex to its right (resp. left). A pal is good if it is defective or in a lonely barrier.

(R2) Every border $C$-vertex $x$ whose central neighbour is not in $C$ sends $\epsilon(x) / 2$ to each of its good pals, if it has two of them and $\epsilon(x)$ to its good pal, if it has exactly one.

(R3) Every border vertex $x$ in a right (resp. left) barrier sends $\epsilon(x)$ to its right (resp. left) pal. Every central vertex $x$ of a right (resp. left) barrier sends to its right (resp. left) pal $\epsilon(x)$ if it is in $B_{2}$ and $\frac{1}{18}$ if it is in $B_{1}$.

(R4) Every generous $B_{1}$-vertex not in a barrier sends $\frac{1}{36}$ to each of its pals.

(R5) Every central $B_{1}$-vertex whose left (resp. right) neighbour is not in $C$ sends $\frac{3}{18}$ to its right (resp. left) neighbour.

(R6) Every border $B_{2}$-vertex whose central neighbour is in $B_{2}$ and adjacent to a central $B_{3}$ sends $\frac{1}{36}$ to this later vertex.

(R7) Every central $B_{2}$-vertex with a border $C$-neighbour and a central $C$-neighbour sends $\frac{1}{36}$ to its central $C$-neighbour.

(R8) Every central $B_{0}$-vertex or central $B_{1}$-vertex with its two central neighbours in $C$ sends $\frac{1}{18}$ to each of its central neighbours. 
(R9) Every central vertex in a right (resp. left) barrier resend to its right (resp. left) pal all the charge its receives from border vertices to its left (rep. right) by (R2).

(R10) A central $B_{2}$-vertex with a $B_{3}$-neighbour to its right (resp. left) and a $B_{2^{-}}$ neighbour to its left (resp. right) sends $\frac{1}{36}$ and everything it gets from the left (resp. right) to its right (resp. left) neighbour.

It is routine to check that every non-defective vertex sends at most its excess and that its final excess is non-negative. We now consider defective vertices. Let $v=(a, 2)$ be a defective vertex. Let us show that its final excess $\epsilon^{*}(v)$ is non-negative.

We first consider the case when $v$ is in $B_{4}$.

- Assume first that $v$ has three $U_{2}$-neighbours and one neighbour in $U_{3} \cup U_{4}$. Then its original excess $\epsilon(v)$ is at least $1-\frac{7}{18}-3 \times \frac{7}{36}-\frac{7}{54}=-\frac{11}{108}$.

By symmetry, we may assume that $(a, 3) \in U_{2},(a-1,3) \in C$ and $(a+1,3) \in$ $U$. Hence $(a-1,2)$ is in $U_{3} \cup U_{4}$ because $N[(a-1,2)] \cap C \neq N[(a, 3] \cap C$. Thus $(a, 1)$ and $(a+1,2)$ are in $U_{2}$. Since $N[(a, 1)] \cap C \neq N[(a+1,2] \cap C$, $(a-1,1)$ and $(a+2,2)$ are in $C$ and $(a+1,1) \in U$. But $(a+1,1)$ and $(a+1,3)$ must have a neighbour in $C$, so $(a+2,1)$ and $(a+2,3)$ are in $C$. Hence the column of index $a+2$ is a left barrier. So $v$ receives at least $3 \times \frac{1}{36}$ by (R3) from the vertices of this barrier and $\frac{1}{7^{2}}$ from each of $(a-1,1)$ and $(a-1,3)$ by $(\mathrm{R} 2)$. Hence $\epsilon^{*}(v) \geq-\frac{11}{108}+3 \times \frac{1}{36}+2 \times \frac{1}{72}>0$.

- Assume now that $v$ has two $U_{2}$-neighbours and two neighbours in $U_{3} \cup U_{4}$. Then its original excess $\epsilon(v)$ is at least $1-\frac{7}{18}-2 \times \frac{7}{36}-2 \times \frac{7}{54}=-\frac{1}{27}$. Observe that $(a, 1)$ and $(a, 3)$ may not both be in $U_{3}$ for otherwise $(a-1,2)$ and $(a+1,2)$ would also be in $U_{3}$. Hence without loss of generality, we are in one of the following two subcases:

- $\{(a-1,1),(a-1,3),(a+1,3)\} \subseteq C$ and $(a+1,1)$ is in $U$. Then $(a+2,1)$ must be in $C$. Hence the three vertices $(a-1,1),(a-1,3),(a+1,3)$ send each $\frac{1}{72}$ to $v$ by (R2). Hence $\epsilon^{*}(v) \geq-\frac{1}{27}+3 \times \frac{1}{72}>0$.

- $\{(a-2,2),(a-1,3),(a+1,1),(a+2,2)\} \subseteq C$ and $(a-1,1)$ and $(a+1,3)$ are in $U$. Then $(a-2,1)$ and $(a+2,3)$ must be in $C$. Observe that the columns of index $a-2$ and $a+2$ are not barriers since $(a-1,1)$ and $(a+1,3)$ are in $U_{1}$ and $(a-1,3)$ and $(a+1,1)$ are not in $U_{1}$. If $(a-2,2)$ is not defective, then $(a-1,3)$ sends at least $\frac{1}{36}$ to $v$ by (R2) and $(a+1,1)$ sends at least $\frac{1}{72}$ to $v$ by (R2). Hence $\epsilon^{*}(v) \geq-\frac{1}{27}+\frac{1}{36}+\frac{1}{72}>0$. If $(a-2,2)$ is defective, then it is in $B_{3}$. Since the code is identifying $(a-3,1)$ is in $C$, so $(a-2,1)$ is a border $B_{1}$-vertex. So it sends $\frac{1}{36}$ to $v$ by (R4). As $v$ receives at least $\frac{1}{72}$ from each of $(a-1,3)$ and $(a+1,1)$, we have $\epsilon^{*}(v) \geq-\frac{1}{27}+\frac{1}{36}+2 \times \frac{1}{72}>0$.

We now consider the case when $v \in B_{3}$. Let $w$ be its $C$-neighbour. $w$ is a central vertex, for otherwise $w$ would be a generous vertex by Proposition 5 and thus $v$ would not be defective. By symmetry, we may assume that $w=(a-1,2)$. 
- Assume first that $v$ has one $U_{1}$-neighbour and two $U_{2}$-neighbours. Up to symmetry, the $U_{1}$-neighbour $z$ is either $(a+1,2)$ or $(a, 3)$.

- If $z=(a+1,2)$, then $(a+1,1)$ and $(a+1,3)$ are in $U$ and so $(a-1,1)$ and $(a-1,3)$ are in $C$. Hence $w$ is in $B_{1}$ because a defective vertex has no generous neighbour. Thus $w$ sends $\frac{3}{18}$ to $v$ by (R5). So $\epsilon^{*}(v) \geq 0$.

- Assume that $z=(a, 3)$. Then $(a-1,3)$ and $(a+1,3)$ are in $U$. Moreover $(a+1,2)$ and $(a, 1)$ are in $U_{2}$ and so $(a+1,1)$ is not in $C$. It follows that $(a-1,1),(a+2,2),(a+2,1)$ and $(a+2,3)$ are in $C$. The column of index $a+2$ is a left barrier.

We claim that $v$ receives at least $\frac{2}{18}$ from its right and at least $\frac{1}{18}$ from its left. This yields $\epsilon^{*}(v) \geq 0$.

Let us show that $v$ receives $\frac{2}{18}$ from its right. If one vertex of the column of index $a+2$ is in $B_{1}$, the vertices of the barrier send at least $\frac{1}{18}+2 \times \frac{1}{36}=$ $\frac{2}{18}$ to $v$. Hence we may assume that $(a+3,1),(a+3,2)$ and $(a+3,3)$ are in $U$. Furthermore by Proposition $1,(a+3,1)$ and $(a+3,3)$ are in $U_{2}$ so $(a+4,1)$ and $(a+4,3)$ are in $C$. If $(a+4,2)$ is in $C$, then $(a+3,2) \in U_{2}$ and so $\epsilon((a+2,2))=\frac{2}{9}$. Hence $v$ receives at least $\frac{2}{9}+2 \times \frac{1}{36}>\frac{2}{18}$ from its right. If $(a+4,2)$ is not in $C$, then by (R2) $(a+4,1)$ and $(a+4,3)$ send in total $\frac{1}{36}$ to $(a+2,2)$ which redirect it to $v$ by (R9). In addition, the barrier send at least $3 \times \frac{1}{36}$ to $v$ by (R3). Hence $v$ receives at least $\frac{2}{18}$ from its right.

Now, either $(a-1,2)$ is in $B_{1}$ in which case it sends $\frac{1}{18}$ to $v$ by (R8), or $(a-1,2)$ is in $B_{2}$ and sends $\frac{1}{36}$ to $v$ by $(\mathrm{R} 7)$ and $(a-1,1)$ is in $B_{2}$ or $B_{1}$ and sends $\frac{1}{36}$ to $v$ by (R6) or (R4). In both cases, $v$ receives $\frac{1}{18}$ from its left.

- Assume that $v$ has one $U_{1}$-neighbour, one $U_{2}$-neighbour and one neighbour in $U_{3} \cup U_{4}$. Then $\epsilon(v) \geq 1-\frac{7}{18}-\frac{7}{18}-\frac{7}{36}-\frac{7}{54}=-\frac{11}{108}$. Let $t$ be the neighbour of $v$ in $U_{3} \cup U_{4}$. By symmetry, we may assume that $t=(a, 3)$ or $t=(a+2,2)$.

- Assume that $t=(a, 3)$. Then $(a-1,3),(a+1,3)$ are in $C$ and $(a-1,1)$, $(a+1,1)$ and $(a+2,2)$ are in $U$. Thus $(a+2,1)$ is in $C$.

If $(a-1,2)$ is in $B_{1}$, then it sends $\frac{1}{18}$ to $v$ by (R8). If not $(a-1,2)$ is in $B_{2}$ and thus sends $\frac{1}{36}$ to $v$ by (R7). Moreover $(a-1,3)$ is in $B_{1} \cup B_{2}$ and thus sends $\frac{1}{36}$ by (R4) or (R6). Hence $v$ receives at least $\frac{1}{18}$ from its left. Let us now show that $v$ receives at least $\frac{5}{54}$ from its right. Since $N[(a+$ $1,1)] \cap C \neq N[(a+2,1)] \cap C$, we have $(a+3,1) \in C$. Since $N[(a+1,3)] \cap$ $C \neq N[(a+2,3)] \cap C$, we have $(a+3,3) \in C$. If $(a+3,2)$ is in $C$, then this vertex is not good. So $(a+1,3)$ sends all its excess to $v$ by (R2). This excess is at least $\frac{5}{54}$. If $(a+3,2)$ is not in $C$, then $(a+2,3)$ is in $C$, because $N[(a+2,2)] \cap C \neq N[(a+1,1)] \cap C$. Hence $(a+2,3)$ is in $B_{1}$ and it is not generous. So its excess is at least $\frac{2}{9}$ and by (R2), it sends at least $\frac{1}{9}$ to $v$.

Hence $v$ receives at least $\frac{1}{18}$ from its left and $\frac{5}{54}$ from its right. Thus $\epsilon^{*}(v) \geq-\frac{11}{108}+\frac{1}{18}+\frac{5}{54}>0$.

- Assume that $t=(a+1,2)$. By symmetry, we may assume that $(a, 3) \in U_{1}$. Then $(a-1,3),(a+1,3)$ and $(a-1,1)$ are in $U$ and $(a+1,1),(a+2,2)$ are in $C$. 
Since $N[v] \cap C \neq N[w] \cap C$, necessarily $(a-2,2) \in C$. Since $(a-1,1)$ and $(a-1,3)$ have different closed neighbourhoods, then a vertex $y$ in $\{(a-2,1),(a-2,3)\}$ is in $C$. If $(a-2,2)$ is in $B_{0}$, then it sends $\frac{1}{18}$ to $w$ by (R8) ; if $(a-2,2)$ is in $B_{1}$, then it sends at least $\frac{1}{18}$ to $w$ by (R5) or (R8); if $(a-2,2)$ is in $B_{2}$, then $(a-2,2)$ sends $\frac{1}{36}$ to $w$ by (R7). In any case, $w$ receives at least $\frac{1}{36}$ from $(a-2,2$,$) which it redirects to v$ with an additional $\frac{1}{36}$ by (R10). So $w$ sends at least $\frac{1}{18}$ to $v$.

Now $(a+1,1)$ has excess at least $\frac{5}{54}$ since it is adjacent to no $U_{1}$ and at least one $U_{3}$. Hence it sends at least $\frac{5}{108}$ to $v$ by $(\mathrm{R} 2)$.

Thus $\epsilon^{*}(v) \geq-\frac{11}{108}+\frac{1}{18}+\frac{5}{108}=0$.

- Assume that $v$ has one $U_{1}$-neighbour and two neighbours in $U_{3} \cup U_{4}$. Then $\epsilon(v) \geq 1-\frac{7}{18}-\frac{7}{18}-2 \times \frac{7}{54}=-\frac{1}{27}$.

Without loss of generality, $(a-1,3),(a+1,3)$ and $(a+2,2)$ are in $C$, and $(a-1,1),(a+1,1)$ are in $U$. Then the vertex $(a+1,3)$ had excess at least

$\frac{17}{108}$ since it is adjacent to two $U_{3}$ and no $U_{1}$. Thus by (R2) it sends at least $\frac{17}{216}$ to $v$. So $\epsilon^{*}(v) \geq-\frac{1}{27}+\frac{17}{216}>0$.

Hence at the end, all the $C$-vertices have non-negative final excess and final weight at least $\frac{7}{18}$. This finishes the proof of Theorem 4 .

\section{References}

1. Y. Ben-Haim and S. Litsyn. Exact minimum density of codes identifying vertices in the square grid. SIAM J. Discrete Math.: 69-82, 2005.

2. I. Charon, I. Honkala, O. Hudry, and A. Lobstein. General bounds for identifying codes in some infinite regular graphs. Electron. J. Combin. 8 (1), Research Paper 39, 21 pp. , 2001.

3. G. Cohen, S. Gravier, I. Honkala, A. Lobstein, M. Mollard, C. Payan, and G. Zémor. Improved identifying codes for the grid, Comment to [4]

4. G. Cohen, I. Honkala, A. Lobstein, and G. Zémor. New bounds for codes identifying vertices in graphs. Electronic Journal of Combinatorics, 6(1) R19, 1999.

5. A. Cukierman and G. Yu. New bounds on the minimum density of an identifying code for the infinite hexagonal grid Discrete Applied Math. 161:2910-2924, 2013.

6. M. Daniel, S. Gravier, and J. Moncel. Identifying codes in some subgraphs of the square lattice. Theoretical Computer Science 319:411-421, 2004.

7. I. Honkala. A family of optimal identifying codes in $\mathbb{Z}^{2}$. J. Combin. Theory Ser. A 113(8):1760-1763, 2006.

8. V. Junnila. New lower bound for 2-identifying code in the square grid. Discrete Appl. Math. 161(13-14):2042-2051, 2013.

9. V. Junnila and T. Laihonen. Optimal lower bound for 2-identifying codes in the hexagonal grid. Electron. J. Combin. 19 (2), Paper 38, 2012.

10. M. Karpovsky, K. Chakrabarty, and L. B. Levitin. On a new class of codes for identifying vertices in graphs. IEEE Trans. Inform. Theory 44:599-611, 1998.

11. S. Ray, D. Starobinski, A. Trachtenberg, and R. Ungrangsi. Robust location detection with sensor networks. IEEE Journal on Selected Areas in Communications 22(6):1016-1025, 2004.

12. P. J. Slater. Fault-tolerant locating-dominating sets. Discrete Math. 249(1-3):179189, 2002. 